\title{
What foods are Northern Ireland supermarkets promoting? A content analysis of supermarket online.
}

\author{
R.K. Price ${ }^{1}$, M.B. Livingstone ${ }^{1}$, A.A. Burns ${ }^{2}$, S. Furey ${ }^{2}$, U. McMahon-Beattie ${ }^{2}$ and \\ L.E. Holywood ${ }^{2}$ \\ ${ }^{1}$ Northern Ireland Centre for Food and Health, Ulster University, Cromore Road, Coleraine, N.Ireland. BT52 ISA and \\ ${ }^{2}$ Ulster Business School, Ulster University, Cromore Road, Coleraine, N.Ireland. BT52 ISA
}

Price promotions are widely used by supermarkets to encourage purchase of targeted products more quickly, more frequently, and/or in greater quantities. These promotions have been shown to be effective in altering consumer behaviour, albeit in the short-term ${ }^{(1,2)}$. US research has shown that price promotions favour processed, energy dense foods ${ }^{(3)}$. On the other hand, price promotions have been successful in increasing the sales of healthier food ${ }^{(4-6)}$ suggesting that appropriately targeted pricing strategies could be used effectively to improve diet patterns. The aim of this study was to conduct a content analysis of online 'Top Offers' promoted by supermarkets across Northern Ireland (NI).

Food promotions $(n=1885$; food $(n=1613,86 \%)$, beverages $(n=272,14 \%)$; Branded $(n=1407,75 \%)$, Own-brand products $(\mathrm{n}=478,25 \%))$ from the 'Top Offer' section of leading NI supermarkets (Tesco, ASDA, and Sainsbury's) and a convenience store (SPAR) were collected online every 3 weeks between April 2014 and April 2015 (12 months; 18 data collections). For each food product promoted the energy and nutrient information was obtained (per $100 \mathrm{~g} / 100 \mathrm{ml}$ ) and the healthiness assessed using 1.) Nutrient Quality (NQ) scoring method based on the Food Standard Agency's Front-of-Pack labelling system (focusing on the risk nutrients sugar, salt, fat, saturated fat) and energy cut-offs defined by Bell et al. ${ }^{(7)}$, and 2.) Food Type score, as defined by the sections of Public Health England (PHE)'s Eatwell Plate.

Overall NQ scores for the food items collected was high (mean 10.7 / $15 \pm$ SD 2.9) and the median score was in the high NQ band $(\geqslant 12$ NQ score; $34 \%)$, followed by the medium NQ (8-12 NQ score; $37 \%)$ and lastly the low NQ band (<8 NQ score; $29 \%)$. There was no significant difference between the NQ score obtained by supermarkets and the convenience store $(P=0.405)$, or between branded and own-branded products $(\mathrm{p}=0 \cdot 107)$. Food types promoted differed significantly to the PHE Eatwell recommendations $(\mathrm{P}<0.001$; Pearson Chi-squared value $=744.2)$ and were as follows (current study vs Eatwell recommendations) 'High Fat High Sugar Foods' (33\% vs $7 \%$ ), 'Bread, Rice, Potatoes \& Pasta' (21\% vs 33\%), 'Meat, Fish Eggs and Beans' (20\% vs $12 \%)$, 'Fruit and Vegetables' (14\% vs $33 \%)$, 'Milk and Dairy Products' (12\% vs $15 \%)$.

In contrast to the popular perception that food promotions favour less healthy foods, findings in the current study showed that NI supermarkets are promoting a wide range of both healthy (high NQ) and less healthy (low NQ) foods, with the majority of foods falling into the high NQ band. However there was some over-representation of 'High fat High sugar foods' and under-representation of 'Fruit and Vegetables' compared with the PHE Eatwell plate recommendations. More research is needed to investigate how findings within the present study impact on consumer behaviour and food intake.

This work was funded by the Food Safety Authority in Northern Ireland (FSANI) and the Consumer Council for Northern Ireland, Project FS305021.

1. Hawkes C (2009) Nutr Rev 67, 333-342.

2. Hamlin RP, Lindsay S, Insch A (2012) Appetite. 58, 256-264.

3. Ethan D, Samuel L, Basch CH (2013) J. Community Health 38, 521-528.

4. French (2003) J Nutr 133, 841S-843S

5. Waterlander WE, Steenhuis IHM, de Boer MR et al. (2012) Int J Behav Nutr Phys Act 9, 11.

6. Waterlander WE, Steenhuis IHM, de Boer MR et al. (2013) Int J Behav Nutr Phys Act 10, 59.

7. Bell E, Castellanos V, Pelkman C et al. (1998) Am J Clin Nutr 67, 412-420. 